\title{
Impact of Cognitive Radio on Radio Astronomy
}

\section{M.J. Bentum ${ }^{1}$}

ASTRON, University of Twente

Dwingeloo, Enschede,

The Netherlands

E-mail:m.j.bentum@utwente.n1

\section{A.J. Boonstra}

ASTRON

E-mail: boonstra@astron.nl

\section{W.A. Baan}

ASTRON

E-mail: baan@astron.nl

The introduction of new communication techniques requires an increase in the efficiency of spectrum usage. Cognitive radio is one of the new techniques that fosters spectrum efficiency by using unoccupied frequency spectrum for communications. However, cognitive radio will increase the transmission power density and cause an increasing level of Radio Frequency Interference (RFI), which may impact other services and particularly passive users of the spectrum. In this paper we present the principles of cognitive radio and introduce a model for its impact on radio astronomy.

RFI mitigation workshop Groningen, the Netherlands March 29-31, 2010

\footnotetext{
$1 \quad$ Speaker
} 


\section{Introduction}

The number of applications for wireless systems is increasing steadily and rapidly, not only in telecommunication systems, but also in new application areas such as wireless sensor networks and body-area networks. All of these applications co-exist in parallel and compete for the use of limited available spectral resources. The success of wireless communication is likely to become a hazard to itself, resulting in increasing mutual interference of radio devices and spectral congestion. The vision of the Wireless World Research Forum [1] is that of 7 trillion wireless devices serving 7 billion people by 2017. In this vision everyone will be served by wireless devices. These are affordable to purchase and operate. The technology behind them is invisible to the users, as it is based on machine-to-machine communications; it includes sensors and tags (e.g. in transport and weather systems), and it will provide ambient intelligence and context sensitivity as all devices are part of the (mobile) internet.

Due to the increasing number of users and the associated bandwidth requirements of these devices, new adaptive spectrum sharing and spectrum re-use models have to be designed, and the spectrum efficiency of future wireless radio systems needs to be increased.

Existing techniques for spectrum sharing, such as the introduction of unlicensed bands (ISM-bands), using underlay in licensed bands (like Ultra Wide Band), opportunistic sharing, and recycling, all exploit the SINR margin of legacy systems and spatial multiplexing and beamforming.

The drawbacks of these existing techniques are that they operate without any knowledge or sense of spectrum occupancy or availability. They only deploy limited adaptability to the spectrum environment and most of the time use pre-determined fixed parameters such as the bandwidth, center frequency, packet length, synchronization, coding and protocols. A new radio philosophy is to make all of these parameters adaptive: this is the import of the drive towards cognitive radio technology.

Although a more efficient use of the spectrum is needed for fulfilling future needs, this increase in usage will impact passive users of the spectrum, such as radio astronomy. In [2] we discussed the co-existence between cognitive radio and radio astronomy in more detail. In this paper we will present the impact of radio systems, in particularly cognitive radio systems, on radio astronomical instruments.

\section{Principles of Cognitive Radio (CR)}

Some licensed communication frequency bands are used very inefficiently. Recent reports conclude that the licensed frequency bands are only used on average $10 \%$ of the time. Clearly the utilization of the spectrum can be improved by permitting secondary users access to the spectrum. So such users need to sense current activity in the spectrum, and, if it is not used, be permitted to use it for new wireless communication. This requires a current awareness of the spectrum, and an ability to adapt its inserted transmission pattern and frequency use accordingly on a non-interference basis. This operational mode is referred to as Cognitive Radio (CR), and was originally proposed by Mitola [3]. This concept of CR is based on the assumption that if no 
transmission is detected in a frequency band, the band is unused. However, services that are always in receiving mode cannot be detected by the spectrum sensing electronics of cognitive radios founded on this principle. Besides radio astronomy, other passive as well as some active applications may suffer from this approach. In the next section we address this situation and calculate the levels of in-band signals for radio astronomy observations.

In summary, we use the following definition of cognitive radio: Cognitive radio is a paradigm for wireless communication in which either a network or a wireless node changes its transmission or reception parameters to communicate efficiently to avoid interfering with other licensed, and unlicensed users.

\section{Interference for radio astronomy}

Interference levels are considered to be harmful to the Radio Astronomy Service when the rms fluctuations of the system noise at the receiver output increase by $10 \%$ or more due to the presence of interference. In the International Telecommunication Union (ITU) Recommendation ITU-R RA.769-2 the spectral power flux thresholds for interference in the allocated Radio Astronomy Services (RAS) frequency band is listed using a reference integration time of 2000 sec.

Every spectral band is of interest for radio astronomy research, and the ITU-R RA.769-2 thresholds are required for their protection from CR. In [2] we showed that coexistence of cognitive radio and radio astronomy in the RAS bands is not possible. This is also the case for the active bands that are used by the RAS on a no-interference and no-protection basis. We can distinguish two types of interference for the case of observing outside the protected RAS bands:

- In-band interference. This source of interference results from signals in the same frequency band as the radio astronomy observations. This may be generated by other spectrum users, and also by electronic equipment. Interference is noticeable when power levels are similar to or higher than those of radio astronomical sources. If the interference levels are very high, the gain of the very sensitive, broadband, analogue front-end of the radio astronomical instrument may become non-linear and cause gain compression to occur.

- Adjacent band interference - including Out-of-Band interference and Intermodulation. Interference due to several signals entering the analogue front-end simultaneously. This is noticeable when the analogue front-end becomes non-linear.

Let us first assume free-space propagation of transmitted signals to the radio astronomical instrument. The received power of an interferer is then calculated as:

$$
P_{r}=S . A_{e f f}=\frac{P_{t} G_{t}}{4 \pi R^{2}} \cdot G_{t} \cdot \frac{\lambda^{2}}{4 \pi}
$$


with $P_{r}$ being the received power, $P_{t}$ the transmitted power of the interferer, $G_{t}$ and $G_{r}$ the transmitter and receiver gain respectively, and $\mathrm{R}$ the distance to the transmitter. When both the transmitting and receiving antennas are dipoles, this can be simplified to

$$
P_{r}=P_{t} \cdot 1.64^{2}\left(\frac{\lambda}{4 \pi R}\right)^{2}
$$

This is a reasonable approximation for the RAS receiving antenna as it would expect to receive the interference signal in the sidelobes of its antenna. For example, lets assume free- space propagation for a $1 \mathrm{GHz}$ interferer at a distance of 10 kilometers, with an ERP of $1 \mathrm{~W}$, and a bandwidth of $10 \mathrm{MHz}$. This gives a power flux density of $-178 \mathrm{dBWm}^{-2} \mathrm{~Hz}^{-1}$. In the ITU-R RA.769-2 the threshold for a $10 \%$ increase of rms fluctuations of the system noise at the receiver output is used. We use this criterion as the threshold for calculating the acceptable power flux density for observations outside the protected RAS bands. The threshold values at 1 $\mathrm{GHz}$ are $-251 \mathrm{dBWm}^{-2} \mathrm{~Hz}^{-1}$ for spectral lines and $-239 \mathrm{dBWm}^{-2} \mathrm{~Hz}^{-1}$ for continuum observations, a difference of 60 to $73 \mathrm{~dB}$ !

For a cognitive radio system with a transmit power of $1 \mathrm{nW}$ and a bandwidth of $10 \mathrm{MHz}$, the distance to the radio astronomical observatory, without exceeding the ITU-R RA.769 threshold is

$$
R=\frac{1,64}{4 \pi} \cdot \lambda \cdot \sqrt{\frac{P_{t}}{P_{r}}} \Rightarrow R=1050 \mathrm{~km}
$$

Of course this is only a theoretical number, since free-space propagation is used. ITU-R P.15464 [8] describes a method for point-to-area radio propagation prediction for terrestrial services in the frequency range $30 \mathrm{MHz}$ to $3000 \mathrm{MHz}$. Using this recommendation, a more accurate estimate can be obtained. In Figure 1 the curves represent field strength as a function of distance for a $1 \mathrm{~kW}$ effective radiated power (ERP) at a variety of different transmitting antenna heights with respect to the receiving antenna height for a $600 \mathrm{MHz}$ frequency. The curves represent exceeded field-strength values $50 \%$ of the time on a land path. This figure can be used to determine the necessary separation distance for a $1 \mathrm{nW}$ transmitter to not exceed the RAS threshold value. The horizontal line, with the attenuation for free-space propagation loss for a 1050 kilometer distance, results in needed separation distances for the various heights. In the case of a height of 10 meters, the threshold value is obtained at a distance of less than about 10 $\mathrm{km}$. 


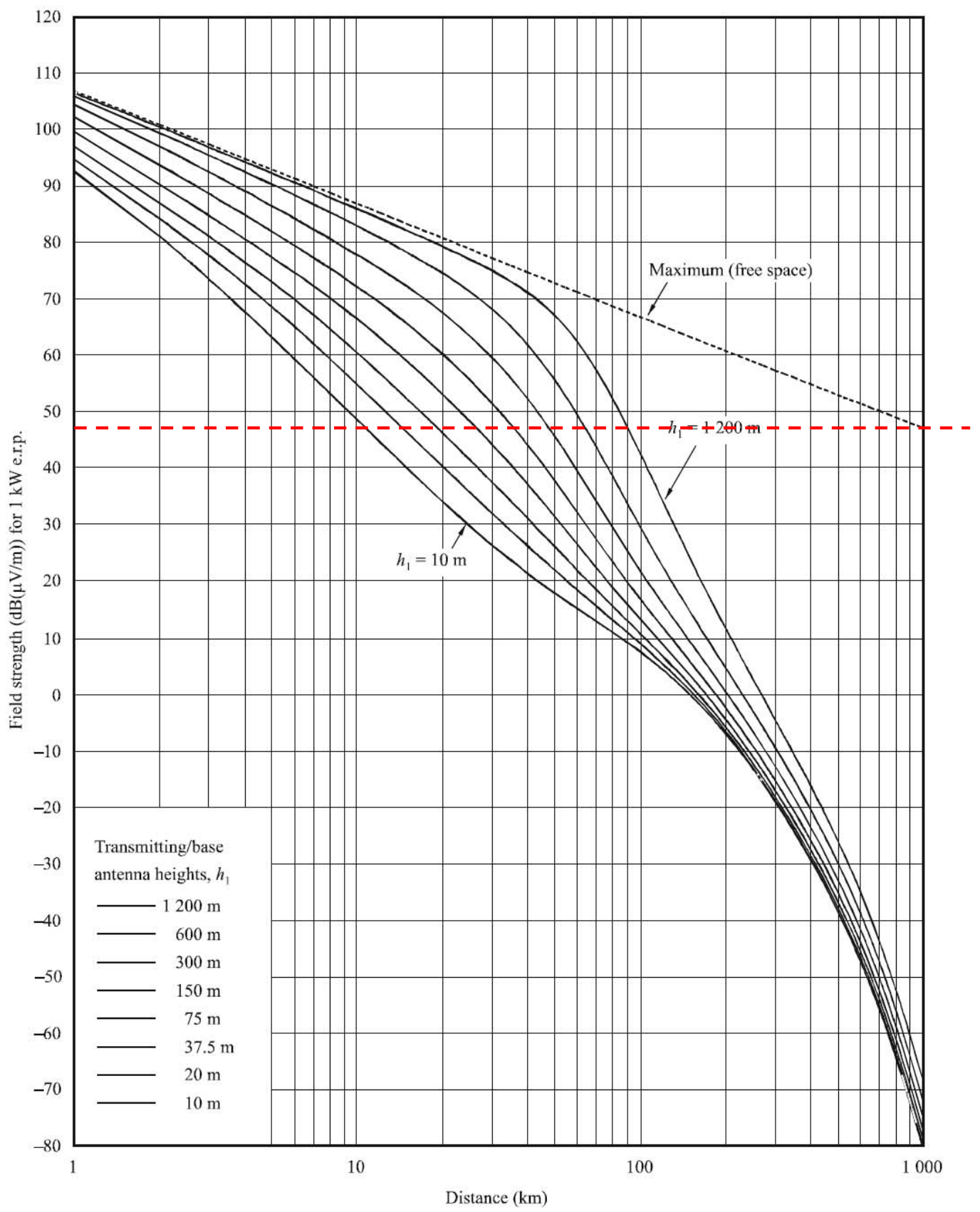

Figure 1: Field strengths of a transmitter of 1kW ERP for various antenna heights [8].

The signal strength must be larger to introduce blocking interference and inter-modulation products. Let us assume a $1 \mathrm{~dB}$ compression value in the frontend of $20 \mathrm{dBm}$. At this level, phase distortion already occurs, which is not acceptable for radio astronomical observations. At a distance of a kilometer from the radio astronomical instrument we obtain harmful blocking signal powers of about $40 \mathrm{dBm}$. This signal level corresponds to a normal, mobile, radiotransmit power level. 


\section{Co-existence of cognitive radio and radio astronomy}

Several scenarios are considered in this section to determine the impact of cognitive radio on radio astronomy: namely cognitive radio transmitters in passive (protected) bands, radio observations in active bands, and out-of-band emission in the passive band.

\subsection{Scenario 1 - In-band cognitive radio in a passive band}

The threshold calculations needed for useful astronomical observations were presented in section 3. It is easily concluded that almost all possible interferers will show up in astronomical data. For example, a system with a maximum transmit power of $1 \mathrm{nW}$ requires a separation distance of about $10 \mathrm{~km}$. Thus the in-band co-existence of cognitive radio and radio astronomy is not possible. That means that passive radio-astronomy bands must be excluded from use by cognitive radio.

\subsection{Scenario 2 - Passive user in adjacent active band}

The bandwidth used for radio astronomy observations is often much larger than the protected passive band. Observing in active bands will therefore be very challenging. All possible frequencies can (and in future will) be used by CR. As with the previous scenario, the co-existence of CR signals in a specific frequency band and useful astronomical observations is not possible. Therefore RFI mitigation techniques to suppress in-band RFI in the receiver frontend are needed. Examples of such systems are given in [4,5,6]. In the particular case of RFI from cognitive radio systems, the occupied frequency bands are likely to constantly change in time, which requires RAS receivers to be equipped with tuneable filters with a very sharp rolloff, that are provided by low temperature superconducting filters.

The calculations in section 3 show that the filter attenuation should be in the order of 80 to $100 \mathrm{~dB}$ to make observations in active bands possible.

\subsection{Scenario 3- Out-of-band (OOB) emission into the passive band}

A cognitive radio system will sense the surrounding radio environment and will transmit in available unoccupied frequency bands. A common method to implement this functionality is to use multi-carrier based transmission techniques. If the band around a carrier is in use, this band is omitted from the CR transmission. The most used implementation of multi-carrier transmission is OFDM (Orthogonal Frequency Division Multiplexing). One of its disadvantages is poor out-of-band attenuation, which is typically just 15 to $20 \mathrm{~dB}$ for adjacent channels. An additional 60 to $80 \mathrm{~dB}$ of attenuation is needed to reach the maximum acceptable power flux density for the RAS. This can be ensured by large guard bands around the radio astronomy band.

Besides the impact of this poor OOB attenuation on the passive bands, the " free” bands in the rest of the spectrum will be of limited use.

As seen in the previous calculations, in-band interference has the most impact on observations. The receiver front-end must therefore contain adequate bandpass filters to attenuate potential interfering signals effectively. For OOB interference this is done to prevent 
intermodulation products and blocking. In-band suppression can be done by means of tunable filters, for example low temperature superconducting filters.

\section{Conclusions and future research}

Cognitive radio, if used over a broad range of spectrum, constitutes a real threat for radio astronomy. Interference levels are considered to be harmful to the Radio Astronomy Service when the rms fluctuations of the system noise at the receiver output increase by $10 \%$ or more due to the presence of interference. Recommendation ITU-R RA.769-2 gives a list of RAS bands with their protection requirements. Calculations of in-band interference levels of Cognitive Radio systems leads to the conclusion that the portions of the spectrum used for Cognitive Radio applications cannot be used for radio astronomical research. To be able to observe in adjacent frequency bands, the out-of-band emission of Cognitive Radio systems must be 80/100 dB lower in the observation band than current CR systems provide. Higher level of control is needed in the RAS receivers. Indeed special receivers must be used in radio astronomy equipment, to cope with the strong signals in adjacent channels.

\section{References}

[1] M.A. Usitalo. Global vision for the future wireless world from the wwrf. IEEE Vehicular Technology Magazine, 1:4-8, 2007.

[2] M.J. Bentum, A.J. Boonstra and W.A. Baan. The Coexistence of Cognitive Radio and Radio Astronomy, 16th Annual Symposium of the IEEE/CVT, Nov. 19, 2009, Louvainla-Neuve, Belgium

[3] J. Mitola. Cognitive radio: Making software radios more personal. IEEE Personal Communications, 6(4):13-18, 1999.

[4] P.A. Fridman and W.A. Baan. RFI mitigation methods in radio astronomy. Astronomy \& Astrophysics, 378:327-244, 2001.

[5] A.J. Boonstra. Radio Frequency Interference Mitigation in Radio Astronomy. $\mathrm{PhD}$ thesis, Delft University of Technology, ISBN 90-805434-3-8 (www.narcis.info), 2005.

[6] M.J. Bentum, A.J. Boonstra, R.P. Millenaar and A.W. Gunst. Implementation of LOFAR RFI mitigation strategy. General Assembly URSI, August 2008, Chicago, USA.

[7] J. Pezzani. Spurious emissions of a LOFAR station and consequences for the Nancay station. Technical report, l'Observatoire de Paris, Unit Scientific de Nancay, 2009.

[8] ITU. Recommendation ITU-R P11546-4, Method for point-to-area predictions for terrestrial services in the frequency range $30 \mathrm{MHz}$ to $3000 \mathrm{MHz}$. 10-2009. 